\section{Myosin phosphatase dephosphorylates HDAC7, controls its nucleocytoplasmic shuttling, and inhibits apoptosis in thymocytes}

\author{
Maribel Parra, Tokameh Mahmoudi, \\ and Eric Verdin ${ }^{1}$ \\ Gladstone Institute of Virology and Immunology, \\ University of California, San Francisco, \\ San Francisco, California 94158, USA
}

The repressive activity of histone deacetylase 7 (HDAC7), a class IIa HDAC expressed in $\mathrm{CD}^{+} \mathrm{CD}^{+}$ double-positive thymocytes, is regulated by its nucleocytoplasmic shuttling. In resting thymocytes, HDAC7 is nuclear and functions as a transcriptional repressor. After T-cell receptor (TCR) activation, the serine/threonine kinase PKD1 phosphorylates HDAC7, resulting in its nuclear export and the derepression of its target genes. Here, we identify protein phosphatase $1 \beta$ (PP1ß) and myosin phosphatase targeting subunit 1 (MYPT1), two components of the myosin phosphatase complex, as HDAC7-associated proteins in thymocytes. Myosin phosphatase dephosphorylates HDAC7 and promotes its nuclear localization, leading to the repression of the HDAC7 target, Nur 77 , and the inhibition of apoptosis in $\mathrm{CD}^{+} \mathrm{CD8}^{+}$thymocytes.

Supplemental material is available at http://www.genesdev.org.

Received November 22, 2006; revised version accepted February 2, 2007.

Class IIa histone deacetylases (HDACs), which comprise HDAC4, HDAC5, HDAC7, and HDAC9, are highly similar transcriptional repressors that are expressed in a tissue-specific manner (Fischle et al. 1999; Grozinger et al. 1999; McKinsey et al. 2001; Verdin et al. 2003; Yang and Gregoire 2005). The repressive activity of Class IIa HDACs is regulated by signal transduction mechanisms that determine whether they are located in the nucleus or cytoplasm (McKinsey et al. 2001; Verdin et al. 2003; Yang and Gregoire 2005). HDAC7 is highly expressed in $\mathrm{CD} 4^{+} \mathrm{CD} 8^{+}$double-positive thymocytes (Dequiedt et al. 2003). In resting thymocytes, HDAC7 is localized in the nucleus and functions as a transcriptional repressor for the proapoptotic orphan receptor Nur77 and other cellular genes involved in $\mathrm{T}$ lymphocyte differentiation (Dequiedt et al. 2003). After T-cell receptor (TCR) activation or PMA stimulation, the serine/threonine kinase PKD1 phosphorylates HDAC7 on three residues (S155, S318, S448) that are conserved among other class IIa HDACs

[Keywords: HDAC7; myosin phosphatase; nucleocytoplasmic shuttling; thymocytes]

${ }^{1}$ Corresponding author.

E-MAIL everdin@gladstone.ucsf.edu; FAX (415) 355-0855.

Article is online at http://www.genesdev.org/cgi/doi/10.1101/gad.1513107.
(Kao et al. 2001; Dequiedt et al. 2003, 2005; Parra et al. 2005). Phosphorylation of HDAC7 leads to its nuclear export, association with 14-3-3 proteins, and to the derepression of its gene targets, including Nur77 (Kao et al. 2001; Dequiedt et al. 2003, 2005; Parra et al. 2005).

\section{Results and Discussion}

To study the phosphorylation of HDAC7 in vivo, we generated phosphorylation-specific antisera against each of these three residues. Western blotting of immunoprecipitated HDAC7 from a thymocyte hybridoma cell line (DO11.10) stably expressing HDAC7-Flag showed increased HDAC7 phosphorylation at each serine after treatment with PMA (Fig. 1A) or TCR activation via CD3 cross-linking (Fig. 1F). All three residues showed some degree of phosphorylation under basal conditions, consistent with the observation that HDAC7 occurs both in the cytoplasm and nucleus of untreated DO11.10 cells (Fig. 1A; Parra et al. 2005). Phosphatase treatment of immunoprecipitated HDAC7 abolished the reactivity of the different phospho-HDAC7 antibodies, confirming their specificity for phosphorylated HDAC7 (Fig. 1A).

Time-course analysis of HDAC7 phosphorylation after PMA treatment showed that HDAC7 was rapidly phosphorylated at the three conserved serines, reaching a maximum after 1-2 $\mathrm{h}$ of treatment with PMA (Fig. 1B). Unexpectedly, a progressive decrease in phosphorylation was observed for all three residues starting at $4 \mathrm{~h}$ after PMA treatment (Fig. 1B), while the total HDAC7 content did not change (Fig. 1B, $\alpha$-Flag Western blot). To test the possibility that HDAC7 became dephosphorylated by a phosphatase, cells were stimulated with PMA followed by the addition of okadaic acid, a phosphatase inhibitor, for the rest of the time course. Consistent with the hypothesis that HDAC7 is dephosphorylated by a phosphatase, okadaic acid treatment led to persistent phosphorylation of HDAC7 at each site up to $24 \mathrm{~h}$ (Fig. 1B).

To determine whether the changes in HDAC7 phosphorylation affected the subcellular localization of the protein, we followed the fate of an HDAC7-GFP fusion protein in response to PMA. As reported, HDAC7-GFP was present both in the nucleus and cytoplasm under basal conditions and was rapidly excluded from the nucleus after PMA stimulation (Fig. 1C; Dequiedt et al. 2003; Parra et al. 2005). At $2 \mathrm{~h}$ after stimulation, $77.5 \%$ of the cells showed complete exclusion of HDAC7 from the nucleus (Fig. 1C). Significant reimport of HDAC7GFP into the nucleus occurred at $4 \mathrm{~h}$ after PMA treatment, a time course that paralleled HDAC7 dephosphorylation (Fig. 1C,D). By $24 \mathrm{~h},<20 \%$ of the cells showed nuclear exclusion of HDAC7 (Fig. 1C,D). In agreement with these observations, HDAC7 nucleocytoplasmic shuttling correlated with a rapid and transient induction of its target gene, Nur77, peaking at $2 \mathrm{~h}$ and disappearing rapidly thereafter (Fig. 1E). The kinetics of HDAC7 phosphorylation and dephosphorylation were slightly delayed when the cells were stimulated through CD3 cross-linking: HDAC7 became fully phosphorylated at $4 \mathrm{~h}$ after stimulation and was completely dephosphorylated after 8-24 h (Fig. 1F). The delayed kinetics of HDAC7 phosphorylation in response to TCR cross-linking correlated with the maximal induction of 
A

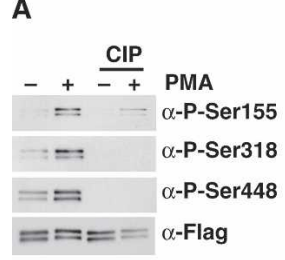

D

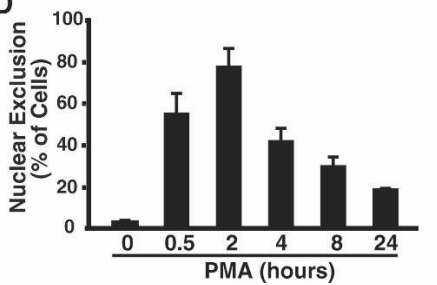

B

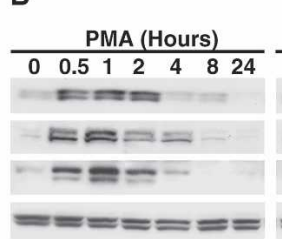

$\mathrm{E}$

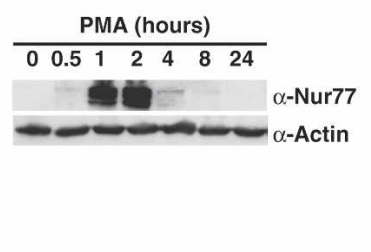

Okadaic Acid

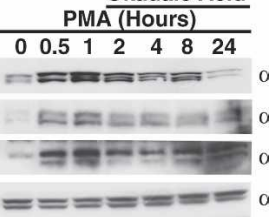

$\alpha-$-P-Ser448 $\alpha$-Flag

$\mathbf{F}$

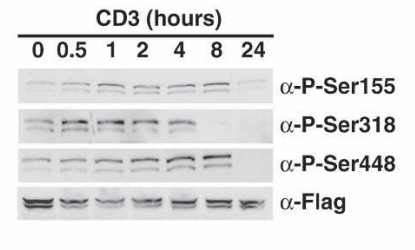

C

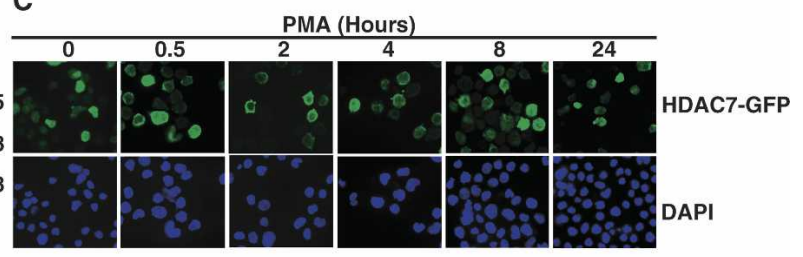

G

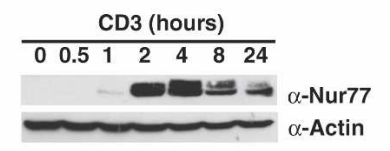

Figure 1. Phosphorylation and nuclear export of HDAC7 is transient after PMA treatment or TCR activation and is reversed by a cellular phosphatase. (A) Western blot showing phosphorylation of Ser155, Ser318, and Ser448 in HDAC7. DO11.10-HDAC7-Flag cells were treated or not with PMA for 30 min. HDAC7 phosphorylation levels were determined by immunoprecipitation of HDAC7-Flag followed by immunoblotting with antisera specific for phosphorylated Ser155, Ser318, and Ser448. The immunoprecipitated material was incubated with CIP before Western blotting where indicated. An anti-Flag Western blot shows equal amounts of HDAC7-Flag were immunoprecipitated. $(B)$ DO11.10HDAC7-Flag cells were treated with PMA alone (left panel), or with PMA + okadaic acid (right panel) for the times indicated. Equal amounts of HDAC7-Flag were immunoblotted with anti-phospho-HDAC7 antisera. $(C)$ Immunofluorescence was performed in DO11.10 cells nucleofected with an HDAC7-GFP expression vector followed $24 \mathrm{~h}$ later by PMA treatment for the indicated times. HDAC7 subcellular distribution was analyzed by immunofluorescence microscopy (representative fields are shown). (D) Quantitation of immunofluorescence microscopy in $C$. The percentage of cells showing nuclear exclusion of HDAC7 is indicated at each time point. One-hundred cells were counted for each point. Error bars represent SEM for four independent experiments. $(E)$ Western blot showing total cell lysates prepared from DO11.10 cells, treated as in $B$, and analyzed by Western blotting with antisera against Nur77 and actin. $(F)$ DO11.10-HDAC7-Flag cells were treated or not with anti-CD3 antibody for the indicated times. HDAC7 phosphorylation was analyzed as in $B .(G)$ Total cell lysates were prepared from DO11.10 cells treated as in $E$ and analyzed by Western blotting with antisera against Nur77 and actin.

Nur77 at $4 \mathrm{~h}$ and its subsequent repression by $24 \mathrm{~h}$ (Fig. $1 \mathrm{G})$. These results indicate that HDAC7 is dephosphorylated by a serine/threonine phosphatase after TCR activation. The activity of this phosphatase leads to HDAC7 nuclear import and repression of Nur77.

To identify a potential HDAC7 phosphatase, we immunopurified HDAC7 from DO11.10 cells stably expressing an HDAC7-Flag protein. The purified HDAC7 complex was subjected to SDS-PAGE and Coomassie staining, followed by mass spectrometry analysis of the HDAC7-associated proteins. We identified protein phosphatase $1 \beta(\mathrm{PP} 1 \beta)$ and the specific PP1 $\beta$ regulatory subunit, myosin phosphatase targeting subunit 1 (MYPT1), as specific HDAC7-interacting proteins (Fig. 2A; Supplementary Information S2). As reported for class IIa HDACs (Grozinger and Schreiber 2000; Wang et al. $2000), 14-3-3$ isoforms $\beta, \varepsilon$, and $\theta$ also coimmunoprecipitated with HDAC7 (Fig. 2A; Supplementary Information S2). The ubiquitously expressed myosin phosphatase complex is composed of PP1 $\beta$, MYPT1, and a third subunit called M20. Myosin phosphatase dephosphorylates myosin light chain (MLC), leading to the relaxation of smooth muscle cells (Ceulemans and Bollen 2004; Ito et al. 2004). MYPT1 is an adaptor protein that mediates the binding of the catalytic subunit, PP1 $\beta$, to the phosphorylated substrate (Ito et al. 2004).

To confirm that myosin phosphatase interacts with HDAC7, we immunoprecipitated HDAC7 from DO11.10 cells expressing HDAC7-Flag and probed for its association with endogenous PP1 $\beta$ and MYPT1. HDAC7 associated with MYPT1 and PP1 $\beta$, but not with other serine/threonine phosphatases, such as $\mathrm{PP} 1 \alpha, \mathrm{PP} 1 \gamma$, PP2A, and PP2B/Calcineurin (Fig. 2B). The 14-3-3 $\beta, \varepsilon$, and $\theta$ isoforms also interacted with HDAC7. The same interactions were also detected between endogenous pro- teins in primary thymocytes: Endogenous PP1 $\beta$, MYPT1, and 14-3-3 proteins coimmunoprecipitated with endogenous HDAC7, but PP1 $\gamma$ did not (Fig. 2C,D). These results demonstrate that myosin phosphatase specifically interacts with HDAC7 in thymocytes.

To test whether PP1 dephosphorylates HDAC7 in vitro, we incubated immunoprecipitated HDAC7-Flag from cells treated or not with PMA with a mixture of recombinant PP1 isoforms $(\alpha, \beta$, and $\gamma)$. Western blot analysis of these HDAC7 preparations with the phosphospecific HDAC7 antisera revealed a complete loss of signal after treatment with PP1 in vitro, demonstrating that PP1 can dephosphorylate HDAC7 (Fig. 3A). To further probe the role of myosin phosphatase in the dephosphorylation of HDAC7 in vivo, we introduced small interfering RNAs (siRNAs) specific for PP1 $\beta$ and MYPT1 into our DO11.10 cells expressing HDAC7. With an siRNA control (siCo), HDAC7 was transiently phosphorylated in response to PMA, with a peak at $2 \mathrm{~h}$, and rapidly dephosphorylated thereafter. In contrast, when siRNAs specific for the myosin phosphatase subunits PP1 $\beta$ and MYPT1 were used, an increase in basal HDAC7 phosphorylation was noted, and HDAC7 remained phosphorylated up to $8 \mathrm{~h}$ after PMA stimulation (Fig. 3B). This result demonstrates that myosin phosphatase specifically dephosphorylates HDAC7 at later time points after PMA stimulation.

Next, we examined the effect of siRNA-mediated knockdown of myosin phosphatase on HDAC7 nucleocytoplasmic shuttling. In cells treated with the control siRNA, HDAC7 was rapidly excluded from the nucleus with a peak at $2 \mathrm{~h}$ after PMA treatment and progressively re-entered the nucleus in a time-dependent manner (Fig. 3D; Supplementary Fig. S3). Importantly, nuclear export was slightly enhanced when myosin phosphatase sub- 

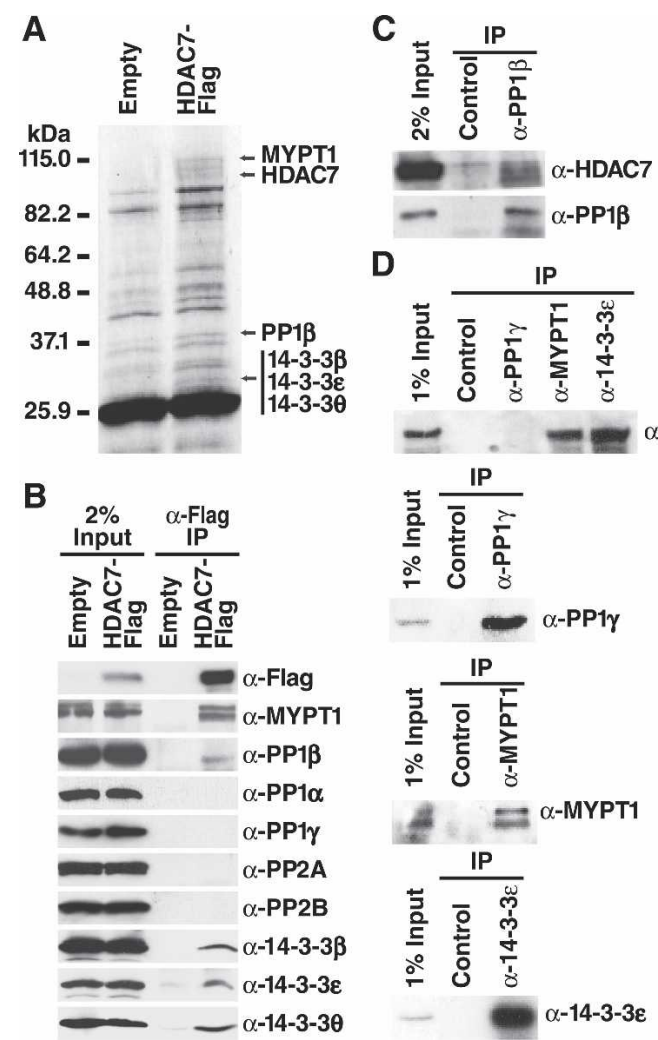

Figure 2. Myosin phosphatase subunits PP1 $\beta$ and MYPT1 coimmunoprecipitate with HDAC7. (A) Coomassie gel of HDAC7-Flagtagged-containing complexes immunoprecipitated from DO11.10 cells with anti-M2 agarose beads. The HDAC7-interacting proteins are indicated by arrows and were identified by mass spectrometry (see Supplemental Material for details). (B) Cell lysates from DO11.10-Empty and DO11.10-HDAC7-Flag cells were immunoprecipitated with anti-M2 agarose beads and analyzed by Western blotting with the indicated antibodies. $(C, D)$ Total cell lysates from mouse primary thymocytes were immunoprecipitated with antibodies against endogenous PP1 $\beta$, PP $1 \gamma$, MYPT1, and 14-3-3e, followed by immunoblotting with anti-HDAC7 antibody. "No $\mathrm{Ab}^{\text {" indicates }}$ no antibody.

units were knocked down, and HDAC7 nuclear re-entry was significantly delayed at later time points (Fig. 3D; Supplementary Fig. S3). These results are in agreement with data showing higher basal phosphorylation and prolonged phosphorylation of HDAC7 after PMA stimulation after knockdown of myosin phosphatase. These observations therefore support the model that HDAC7 is dephosphorylated by myosin phosphatase after TCR activation, leading to its nuclear re-entry.

HDAC7 is recruited to its target promoters via its specific interaction with the transcription factor MEF2D. We have recently conducted a genomic screen of HDAC7 targets and have demonstrated that HDAC7 regulates the transcriptional activity of a cassette of genes that controls thymocyte differentiation and apoptosis (H. Kasler and E. Verdin, in prep.). One of the most highly regulated HDAC7 target is the transcription factor Nur77. To test whether myosin phosphatase regulates Nur77 gene expression, we used nucleofection to introduce siRNAs for PP1 $\beta$ and/or MYPT1 into DO11.10 cells (Fig. 4A). siRNA-transfected cells were activated via TCR cross-linking ( $\alpha$-CD3 antibody), and the expression of Nur77 was analyzed at 24 h, when Nur77 expres- sion is newly suppressed (Fig. 1G). Depletion of PP1 $\beta$, MYPT1, or both resulted in a dramatic superinduction of Nur77 expression after TCR cross-linking (Fig. 4B). Importantly, superinduction of Nur77 expression by knockdown of myosin phosphatase did not occur in cells expressing an HDAC7 mutant that cannot be phosphorylated and is not exported to the cytoplasm in response to TCR activation (HDAC7 $\triangle \mathrm{P}$ ) (Fig. 4B; Dequiedt et al. 2003). These results demonstrate that myosin phosphatase mediates the de novo repression of Nur77 expression by dephosphorylating HDAC7 at late times after TCR activation.

To further analyze the specificity of myosin phosphatase in the regulation of Nur77, we used siRNAs specific for each of the PP1 isoforms, PP $1 \alpha$, PP1 $\beta$, and PP $1 \gamma$ (Fig. $4 C)$. PP $1 \beta$ depletion resulted in the superinduction of Nur77 after TCR activation, whereas depletion of PP1 $\alpha$ or PP1 $y$ had no significant effect (Fig. 4D). Here also, expression of the HDAC7 $\triangle \mathrm{P}$ mutant prevented the superinduction of Nur77 after TCR activation (Fig. 4D).

Nur77 is an orphan nuclear receptor that is rapidly and transiently induced after TCR activation and plays a key role in the induction of negative selection or apoptosis in thymocytes (Liu et al. 1994; Woronicz et al. 1994; Calnan et al. 1995). In addition, many of the proteins recently identified in our screen of genomic HDAC7 tar-
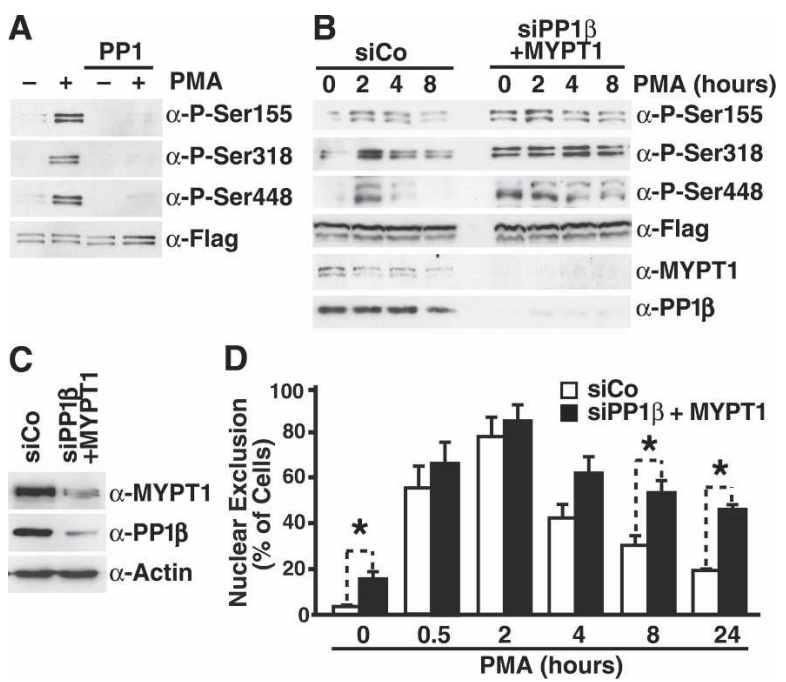

Figure 3. Myosin phosphatase dephosphorylates HDAC7 and induces its nuclear reimport after TCR activation. (A) DO11.10HDAC7-Flag cells were either left untreated or treated with PMA for 30 min. HDAC7 phosphorylation was determined as in Figure 1A. A mixture of recombinant PP1 isoforms was added to the immunoprecipitated material before Western blotting with phosphospecific antisera for HDAC7. (B) DO11.10-HDAC7-Flag cells nucleofected with either siCo or siPP1 $\beta+$ MYPT1 were treated with PMA for the indicated times. HDAC7 phosphorylation was determined as in Figure 1A. HDAC7-Flag, PP1 $\beta$, MYPT1, and actin protein levels are shown. (C) Depletion of PP1 $\beta$ and MYPT1 in DO11.10 cells by siRNA-mediated knockdown. Western blot analysis of PP1 $\beta$ and MYPT1 protein levels $48 \mathrm{~h}$ after nucleofection of control (siCo) or PP1 $\beta$ and MYPT1 (siPP1 $\beta$ + MYPT1) siRNA oligonucleotides. $(D)$ DO11.10 cells were nucleofected with either siCo or siPP1 $1 \beta$ - MYPT1 and with an HDAC7-GFP expression vector. Cells were treated with PMA $24 \mathrm{~h}$ for the indicated periods of time. HDAC7 was localized by immunofluorescence microscopy. The percentage of cells showing nuclear exclusion of HDAC7 is indicated at each time point after PMA addition. One-hundred cells were counted for each point. Error bars represent SEM for four independent experiments. $\left(^{\star}\right) p<0.05$. 
A

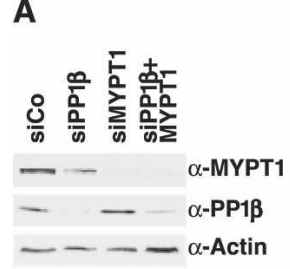

C

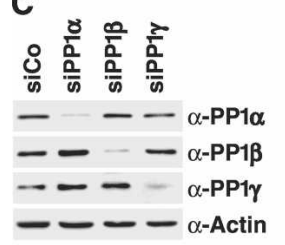

B
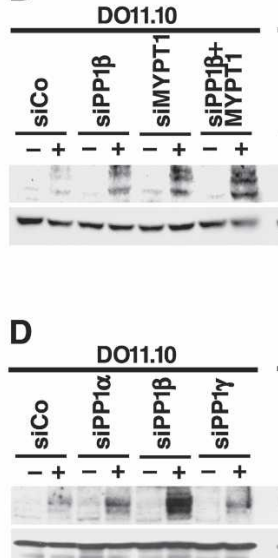
D011.10-HDAC7AP DO11.10-HDAC7AP
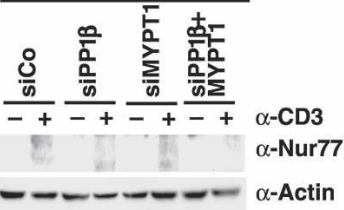

E

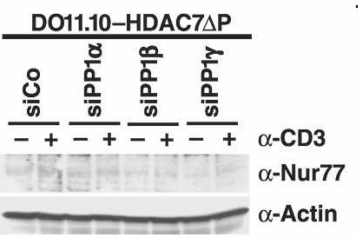

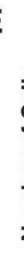
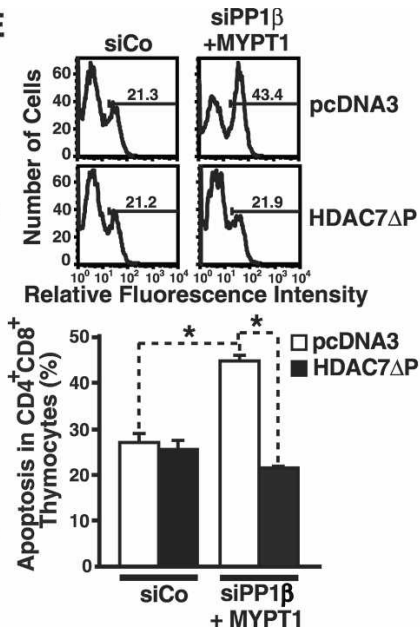

F T-Cell Receptor Complex

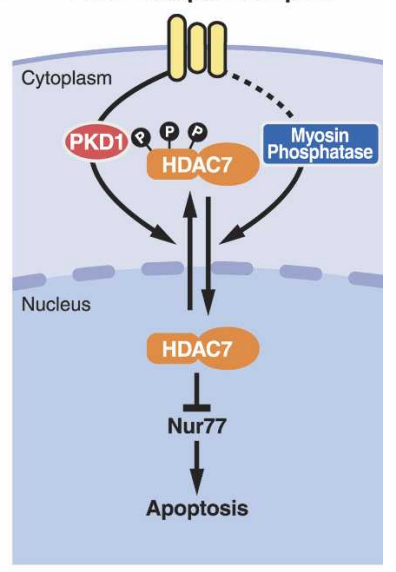

Figure 4. Myosin phosphatase regulates Nur77 activation and thymocyte apoptosis after TCR activation. $(A)$ Depletion of PP1 $\beta$, MYPT1, or both in DO11.10 cells by siRNA-mediated knockdown. PP1 $\beta$ and MYPT1 protein levels were analyzed $48 \mathrm{~h}$ after nucleofection of the different siRNAs. (B) DO11.10-Empty or DO11.10-HDAC7 $\Delta$ P-Flag cells were nucleofected with either siCo, siPP1 $\beta$, siMYPT1, or siPP1 $\beta+$ MYPT1. After $24 \mathrm{~h}$, cells were treated with anti-CD3 antibody and analyzed by Western blotting with antisera against Nur77 and actin. (C) Depletion of PP1 $\alpha$, PP1 $\beta$, or PP1 $\gamma$ in DO11.10 cells by siRNA-mediated knockdown. PP1 $\alpha$, PP1 $\beta$, or PP1 $\gamma$ protein levels were analyzed 48 h after nucleofection of the different siRNAs. $(D)$ DO11.10-Empty or DO11.10-HDAC7 PP-Flag cells were nucleofected with siRNAs for the different PP1 isoforms. Cells were treated and analyzed as in Figure 1A. (E) Primary thymocytes were nucleofected with the indicated siRNAs. Cells were treated with anti-CD3 antibody for $16 \mathrm{~h}$, followed by staining with AnnexinV-APC, anti-CD4-PE, and anti-CD8-FITC. A representative FACS plot is shown that quantifies Annexin V binding ( $X$-axis) in $\mathrm{CD}^{+} \mathrm{CD} 8^{+}$cells. Data are represented as mean \pm SEM of three independent experiments. $\left({ }^{\star}\right) p<0.001$. In the bottom panel, flow histograms illustrate the percentage of apoptotic cells in a representative experiment. $(F)$ Signaling pathways responsible for HDAC7 nucleocytoplasmic shuttling after TCR activation. The nucleocytoplasmic location of HDAC7 is under the competing influences of a kinase (PKD1) and a phosphatase (myosin phosphatase).

gets regulated apoptosis in developing thymocytes $(\mathrm{H}$. Kasler and E. Verdin, in prep.). Accordingly, we tested the role of myosin phosphatase in apoptosis. We nucleofected mouse primary thymocytes with siRNAs specific for PP1 $\beta$ and MYPT1. Apoptosis was measured in $\mathrm{CD} 4^{+} \mathrm{CD} 8^{+}$double-positive thymocytes by flow cytometry and annexin $\mathrm{V}$ binding. In thymocytes treated with a control siRNA, $27 \%$ of double-positive thymocytes were undergoing apoptosis. Knockdown of PP $1 \beta$ and MYPT1 was associated with a significant increase in the rate of apoptosis to $44.7 \%$ (Fig. 4E). Strikingly, expression of the HDAC7 $\mathrm{P}$ mutant suppressed the increased apoptosis measured in thymocytes treated with siRNAs specific for PP1 $\beta$ and MYPT1 (Fig. 4E). This observation demonstrates that myosin phosphatase mediates the survival of thymocytes via dephosphorylation of HDAC7.

Based on the observations described above, we propose that the phosphorylation of HDAC7, its nucleocytoplasmic shuttling, and its activity as a transcriptional repressor in thymocytes are under the competing influences of a protein kinase, PKD1, and a phosphatase, myosin phosphatase (Fig. 4F). During the first $4 \mathrm{~h}$ after cross-linking of the TCR, phosphorylation of HDAC7 is enhanced in response to PKD1 activation (Parra et al. 2005) and, possibly, in response to an inhibition of myosin phosphatase activity. Enhanced phosphorylation of HDAC7 leads to its nuclear export and the derepression of genes, such as Nur77, that are involved in thymocyte apoptosis. Starting at $8 \mathrm{~h}$ after TCR activation, the activity of myosin phosphatase becomes dominant, leading to the dephosphorylation of HDAC7, its re-entry into the nucleus, and the resilencing of Nur77 and other genes that control apoptosis in developing $\mathrm{T}$ cells. The anti-apoptotic role of myosin phosphatase is particularly intriguing with regard to T-cell development in the thymus. Indeed, a frac- tion of developing thymocytes responds to TCR activation by further differentiating into single-positive T cells (CD4 or CD8), a process referred to as positive selection. Our observations support a model in which the level of HDAC7 phosphorylation, controlled by the competing activities of PKD1 and myosin phosphatase, could determine whether the developing $\mathrm{T}$ cells undergo positive or negative selection.

Importantly, myosin phosphatase is also expressed in cardiac and skeletal muscle (Fujioka et al. 1998; Arimura et al. 2001). In cardiomyocytes, overexpression of myosin phosphatase subunits results in the abolition of agonist-induced sarcomere organization, a marker of cardiac hypertrophy (Okamoto et al. 2006). Interestingly, an HDAC5 mutant that cannot be phosphorylated (similar to our HDAC $7 \triangle \mathrm{P}$ ) is refractory to hypertrophic signaling and inhibits cardiomyocyte hypertrophy (Zhang et al. 2002). We speculate that myosin phosphatase could inhibit cardiac hypertrophy by dephosphorylating HDAC5, resulting in its nuclear localization and the repression of specific target genes. Based on the conservation of the sites of phosphorylation in HDAC4, HDAC5, HDAC7, and HDAC9 and on the presence of myosin phosphatase in the tissues where other class IIa HDACs are expressed (e.g., muscle, heart, and CNS), we anticipate that the mechanism illustrated in this report for HDAC7 also contributes to the regulation of other class IIa HDACs in these other tissues.

\section{Materials and methods}

Cells and cell treatment

DO11.10 T cells were grown at $37^{\circ} \mathrm{C}$ in RPMI 1640 supplemented with $10 \%$ fetal bovine serum, $2 \mathrm{mM}$ glutamine, and $50 \mathrm{U} / \mathrm{mL}$ streptomycin/ penicillin. DO11.10 cells stably expressing either empty vector, HDAC7- 
Flag, or HDAC7DP-Flag have been described (Dequiedt et al. 2003). Mouse primary thymocytes were obtained from 4- to 6-wk-old Balb/c mice. Where indicated, PMA was added at a concentration of $10 \mathrm{ng} / \mathrm{mL}$. For CD3 stimulation, tissue culture plates were coated with an anti-CD3 antibody (500A2) at a 1:1000 dilution in PBS overnight at $4^{\circ} \mathrm{C}$.

\section{RNA interference}

Predesigned siRNA pools targeting transcripts of the mouse PP1 $\alpha, \mathrm{PP} 1 \beta$, PP1 $\gamma$, and MYPT1 genes as well as a control siRNA pool were used to knock down the respective genes in DO11.10 cells and mouse primary thymocytes. siRNA control and siRNA MYPT1 were from Dharmacon. The siRNAs for the different PP1 isoforms were from Ambion (for detailed information on the siRNAs, see Supplementary Information S4). siRNAs were delivered by nucleofection (Amaxa).

Nucleofection

DO11.10 cells were transfected using the Amaxa nucleofector kit $\mathrm{R}$ and program O28. Cells were split to $3 \times 10^{5}$ cells $/ \mathrm{mL} 24 \mathrm{~h}$ before nucleofection. Cells $\left(5 \times 10^{6}\right)$ were spun at $1000 \mathrm{rpm}$ for $10 \mathrm{~min}$ at room temperature, resuspended in $100 \mu \mathrm{L}$ of solution $\mathrm{R}$, and nucleofected with $2 \mu \mathrm{g}$ of either siRNA or expression plasmid by program O28. Nucleofected cells were resuspended in $500 \mu \mathrm{L}$ of prewarmed serum-free RPMI lacking antibiotics and allowed to recover for $15 \mathrm{~min}$ at $37^{\circ} \mathrm{C}$ in a $5 \% \mathrm{CO}_{2}$ incubator, and $4.5 \mathrm{~mL}$ of prewarmed complete RPMI was added to the cells. Nucleofection of mouse primary thymocytes was performed with the Mouse T-cell Nucleofector Kit and program X001, following the manufacturer's instructions.

Generation of phospho-HDAC7-specific antibodies

Antisera were raised against mouse HDAC7 phosphorylated at Ser178, Ser344, and Ser479 (Ser155, Ser318, and Ser448 for human HDAC7) after immunization of rabbits with KLH-conjugated peptides (Sigma Genosys). The phosphopeptides used to generate the different antisera were FPLRTV[pSer]EPNLKL for P-Ser178, RPLNRTR[pSer]EPLPPS for P-Ser344, and RPLSRTQ[pSer]SPAAPV for P-Ser479. "[pSer]" indicates the phosphate group on the conserved serines. HDAC7 phospho-specific antibodies were purified from crude rabbit serum by double affinity purification with nonphosphorylated and phosphorylated peptide.

\section{Immunoprecipitation}

Total cellular extracts from DO11.10 cells or primary thymocytes were prepared in PLB buffer $(0.5 \%$ Triton X-100, 0.5 mM EDTA, 1 mM DTT in PBS) supplemented with protease inhibitors (Complete, Roche Molecular Biochemicals). Cellular lysates were precleared with mouse IgGagarose beads (Sigma) for $2 \mathrm{~h}$ at $4^{\circ} \mathrm{C}$. Immunoprecipitations of HDAC7Flag tagged proteins were carried out for $4 \mathrm{~h}$ at $4^{\circ} \mathrm{C}$ with anti-M2 agarose beads (Sigma) at a concentration of $15 \mu \mathrm{L} / \mathrm{mL}$. Immunoprecipitated material was washed three times in IPLS buffer $(50 \mathrm{mM}$ Tris- $\mathrm{HCl}$ at $\mathrm{pH} 7.5$, $0.5 \mathrm{mM}$ EDTA. $0.5 \% \mathrm{NP}-40,150 \mathrm{mM} \mathrm{NaCl}$ ) supplemented with protease inhibitors. For immunoprecipitation of endogenous proteins from primary thymocytes, anti-PP1 $\alpha$, anti-PP1 $\beta$, anti-MYPT1, and anti-14-3-3 antibodies were used at concentrations of $2 \mu \mathrm{g} / \mathrm{mL}$ in combination with a $50 \%$ protein A-Sepharose slurry (Amersham Pharmacia Biotech). Immunoprecipitated material was washed three times in IPLS. Bound proteins were subjected to SDS-PAGE and Western blot analysis.

In vivo HDAC7 phosphorylation

DO11.10-HDAC7-Flag cells were untreated or treated with either PMA or anti-CD3 antibody for the indicated times. Total cellular extracts were prepared in $20 \mathrm{mM}$ Hepes (pH 7.5), $10 \mathrm{mM}$ EGTA, $2.5 \mathrm{mM} \mathrm{MgCl}_{2}, 1 \%$ NP-40, $2 \mathrm{mM}$ orthovanadate, $1 \mathrm{mM}$ dithiothreitol, and $0.5 \mathrm{mM}$ phenylmethyl sulfonyl fluoride supplemented with protease inhibitors, and subjected to Western blot analysis.

In vitro dephosphorylation assays

Lysates were prepared from DO11.10-HDAC7-Flag cells either untreated or treated with PMA for $30 \mathrm{~min}$. Cell lysates were immunoprecipitated and washed as described above. Washed beads were resuspended in $20 \mu \mathrm{L}$ of phosphatase assay buffer $(50 \mathrm{mM}$ Tris- $\mathrm{HCl}$ at $\mathrm{pH} 7.5,0.1 \% 2-\beta$-mercaptoethanol, $0.1 \mathrm{mM}$ EDTA, $1 \mathrm{mg} / \mathrm{mL} \mathrm{BSA}$ ) and treated with $10 \mathrm{U}$ of CIP (New England Biolabs), or $0.5 \mathrm{U}$ of a mixture of recombinant PP1 isoforms (Upstate Biotechnology) was added to the beads. The dephos- phorylation reaction was carried out for $30 \mathrm{~min}$ at $30^{\circ} \mathrm{C}$. The samples were subjected to Western blot analysis.

\section{SDS-PAGE and Western blotting}

SDS-PAGE and Western blot analysis were performed according to standard procedures. Western blots were developed with the ECL detection kit (Amersham Pharmacia Biotech). Anti-PP1 $\alpha$, anti-PP1 $\beta$, and antiPP2A were purchased from Upstate Biotechnology. Anti-PP1 $\gamma$, antiHDAC7, anti-PKD1, anti-PP2B, and anti-14-3-3 were from Santa Cruz Biotechnology. Anti-MYPT1 antibody was from Abcam. Anti-actin antibody was from Sigma. Anti-mouse Nur77 antibody was from BD Pharmingen.

\section{Immunofluorescence}

D011.10 cells $\left(5 \times 10^{6}\right)$ were nucleofected with an HDAC7-GFP expression vector together with either siRNA control or siRNAs for PP1 $\beta$ and MYPT1. Cells $\left(5 \times 10^{5}\right)$ were seeded onto poly-L-lysine-coated coverslips $12 \mathrm{~h}$ after nucleofection and allowed to attach for $12 \mathrm{~h}$. Cells were stimulated with $10 \mathrm{ng} / \mathrm{mL}$ PMA. HDAC7 was localized by immunofluorescence microscopy with a confocal fluorescence microscope (Olympus BX60, Bio-Rad).

\section{Apoptosis assays}

Primary thymocytes were nucleofected with the indicated siRNAs and expression plasmids. Thymocytes were stained with AnnexinV-APC, anti-CD4-PE, and anti-CD8-FITC (BD Pharmingen) $24 \mathrm{~h}$ after nucleofection and analyzed on a FACSCalibur (Becton Dickinson) with CellQuest software. Apoptosis represents the percentage of double-positive thymocytes positive for Annexin V staining.

Statistical analysis

Statistical analyses were performed with SPSS 10.0 (SPSS). Differences between means were assessed by ANOVA, followed by Tukey-Kramer post hoc test.

\section{Acknowledgments}

We thank John C.W. Carroll, Jack Hull, and Chris Goodfellow for graphics, and Stephen Ordway and Gary Howard for editorial assistance. We thank Robert G. Vries, Herb Kasler, Steven Kauder, Brian North, Melanie Ott, Carmen M. Ruiz-Jarabo, and Bjoern Schwer for helpful comments on the manuscript, and Jorge J. Palop and Inma Cobos for help with statistics analysis and for providing reagents. We also thank Drs. Steven C. Hall, H. Ewa Witkowska, and Scott E. Dixon of the Biomolecular Resource Center Mass Spectrometry Facility, and the Sandler Family Foundation, University of California, San Francisco, for their assistance in mass spectrometry acquisition and data analysis. This work was supported by The J. David Gladstone Institutes (E.V.) and by grant number RR 18928-01 from the NIH National Center for Research Resources. M.P. was supported by a post-doctoral fellowship from the Spanish Ministery of Education and Science (MEC). T.M. was supported by a post-doctoral fellowship from the Universitywide Aids Research Program (UARP).

\section{References}

Arimura, T., Suematsu, N., Zhou, Y.B., Nishimura, J., Satoh, S., Takeshita, A., Kanaide, H., and Kimura, A. 2001. Identification, characterization, and functional analysis of heart-specific myosin light chain phosphatase small subunit. J. Biol. Chem. 276: 6073-6082.

Calnan, B.J., Szychowski, S., Chan, F.K., Cado, D., and Winoto, A. 1995 A role for the orphan steroid receptor Nur77 in apoptosis accompanying antigen-induced negative selection. Immunity 3: 273-282.

Ceulemans, H. and Bollen, M. 2004. Functional diversity of protein phosphatase-1, a cellular economizer and reset button. Physiol. Rev. 84: $1-39$.

Dequiedt, F., Kasler, H., Fischle, W., Kiermer, V., Weinstein, M., Herndier, B.G., and Verdin, E. 2003. HDAC7, a thymus-specific class II histone deacetylase, regulates Nur77 transcription and TCR-mediated apoptosis. Immunity 18: 687-698.

Dequiedt, F., Van Lint, J., Lecomte, E., Van Duppen, V., Seufferlein, T., Vandenheede, J.R., Wattiez, R., and Kettmann, R. 2005. Phosphorylation of histone deacetylase 7 by protein kinase $\mathrm{D}$ mediates $\mathrm{T}$ cell 
receptor-induced Nur77 expression and apoptosis. J. Exp. Med. 201: 793-804.

Fischle, W., Emiliani, S., Hendzel, M.J., Nagase, T., Nomura, N., Voelter, W., and Verdin, E. 1999. A new family of human histone deacetylases related to Saccharomyces cerevisiae HDAlp. J. Biol. Chem. 274: 11713-11720.

Fujioka, M., Takahashi, N., Odai, H., Araki, S., Ichikawa, K., Feng, J., Nakamura, M., Kaibuchi, K., Hartshorne, D.J., Nakano, T., et al. 1998. A new isoform of human myosin phosphatase targeting/regulatory subunit (MYPT2): cDNA cloning, tissue expression, and chromosomal mapping. Genomics 49: 59-68.

Grozinger, C.M. and Schreiber, S.L. 2000. Regulation of histone deacetylase 4 and 5 and transcriptional activity by 14-3-3-dependent cellular localization. Proc. Nat1. Acad. Sci. 97: 7835-7840.

Grozinger, C.M., Hassig, C.A., and Schreiber, S.L. 1999. Three proteins define a class of human histone deacetylases related to yeast Hdalp. Proc. Natl. Acad. Sci. 96: 4868-4873.

Ito, M., Nakano, T., Erdodi, F., and Hartshorne, D.J. 2004. Myosin phosphatase: Structure, regulation and function. Mol. Cell. Biochem. 259: 197-209.

Kao, H.Y., Verdel, A., Tsai, C.C., Simon, C., Juguilon, H., and Khochbin, S. 2001. Mechanism for nucleocytoplasmic shuttling of histone deacetylase 7. J. Biol. Chem. 276: 47496-47507.

Liu, Z.G., Smith, S.W., McLaughlin, K.A., Schwartz, L.M., and Osborne, B.A. 1994. Apoptotic signals delivered through the T-cell receptor of a T-cell hybrid require the immediate-early gene nur77. Nature 367 : 281-284.

McKinsey, T.A., Zhang, C.L., and Olson, E.N. 2001. Control of muscle development by dueling HATs and HDACs. Curr. Opin. Genet. Dev. 11: 497-504.

Okamoto, R., Kato, T., Mizoguchi, A., Takahashi, N., Nakakuki, T., Mizutani, H., Isaka, N., Imanaka-Yoshida, K., Kaibuchi, K., Lu, Z., et al. 2006. Characterization and function of MYPT2, a target subunit of myosin phosphatase in heart. Cell. Signal. 18: 1408-1416.

Parra, M., Kasler, H., McKinsey, T.A., Olson, E.N., and Verdin, E. 2005. Protein kinase D1 phosphorylates HDAC7 and induces its nuclear export after T-cell receptor activation. J. Biol. Chem. 280: $13762-$ 13770.

Verdin, E., Dequiedt, F., and Kasler, H.G. 2003. Class II histone deacetylases: Versatile regulators. Trends Genet. 19: 286-293.

Wang, A.H., Kruhlak, M.J., Wu, J., Bertos, N.R., Vezmar, M., Posner, B.I., Bazett-Jones, D.P., and Yang, X.J. 2000. Regulation of histone deacetylase 4 by binding of 14-3-3 proteins. Mol. Cell. Biol. 20: 69046912.

Woronicz, J.D., Calnan, B., Ngo, V., and Winoto, A. 1994. Requirement for the orphan steroid receptor Nur77 in apoptosis of T-cell hybridomas. Nature 367: 277-281.

Yang, X.J. and Gregoire, S. 2005. Class II histone deacetylases: From sequence to function, regulation, and clinical implication. Mol. Cell. Biol. 25: 2873-2884.

Zhang, C.L., McKinsey, T.A., Chang, S., Antos, C.L., Hill, J.A., and Olson, E.N. 2002. Class II histone deacetylases act as signal-responsive repressors of cardiac hypertrophy. Cell 110: 479-488. 


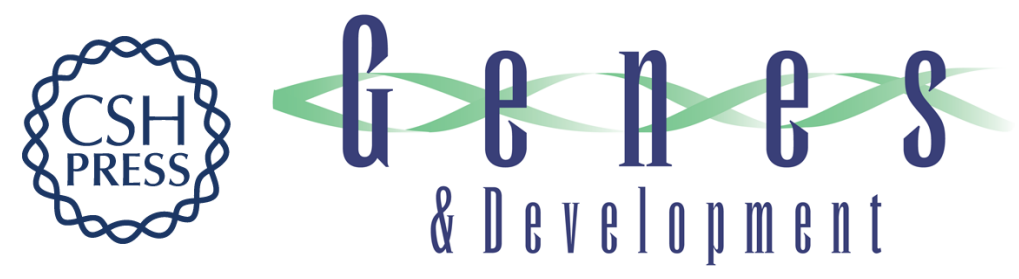

\section{Myosin phosphatase dephosphorylates HDAC7, controls its nucleocytoplasmic shuttling, and inhibits apoptosis in thymocytes}

Maribel Parra, Tokameh Mahmoudi and Eric Verdin

Genes Dev. 2007, 21:

Access the most recent version at doi:10.1101/gad.1513107

Supplemental http://genesdev.cshlp.org/content/suppl/2007/03/09/21.6.638.DC1
Material

References This article cites 20 articles, 9 of which can be accessed free at:

http://genesdev.cshlp.org/content/21/6/638.full.html\#ref-list-1

License

Email Alerting Receive free email alerts when new articles cite this article - sign up in the box at the top

Service

right corner of the article or click here.

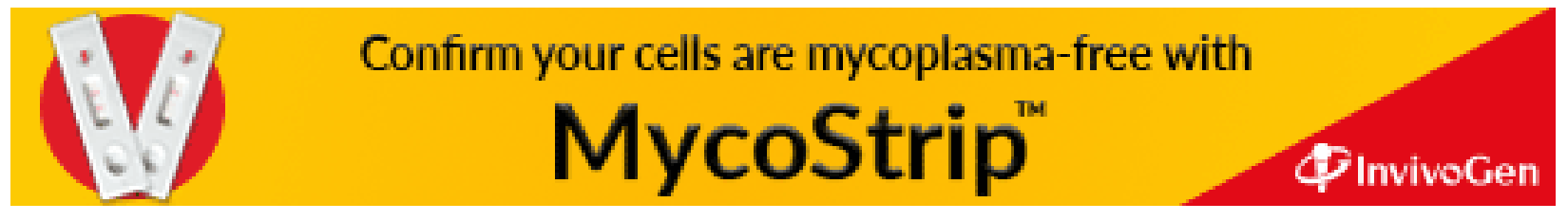

